\title{
SPACE CHARGE EFFECTS IN THE KEK-BOOSTER SYNCHROTRON
}

\author{
Chihiro Ohmori \\ Institute for Nuclear Study, University of Tokyo, Tokyo, Japan
Toshikazu Adachi, Tadamichi Kawakubo, Motohiro Kihara and Isao Yamane National Laboratory for High Energy Physics (KEK), Ibaraki, Japan

Space charge effects have been observed in the KEKPS Booster synchrotron. Beam-profile measurements by the BEAMSCOPE method show that a beam blow-up in the vertical plane occurred during the RF capture process. The intensity dependence of beam blow-up and deformation of beam profile were observed.

\section{INTRODUCTION}

The KEK-PS Booster is a $40-$ to $500-\mathrm{MeV}$ proton synchrotron, which is the injector of the KEK $12 \mathrm{GeV}$ Proton synchrotron, and also delivers a beam to the Booster Synchrotron Utilization Facility (BSF). It is a compact alternating-gradient synchrotron operated at fixed tunes. The repetition rate is $20 \mathrm{~Hz}$ and the synchrotron magnets are excited by a resonance circuit. The linac beam is injected by charge exchange injection and is adiabatically captured in an RF bucket. A bunch is formed in $600 \mu$ s and the bunching factor becomes $\sim 0.3$. A transverse beam blow-up at the early stage of acceleration has been observed [1]. Since the Booster synchrotron was designed for $\mathrm{H}^{+}$multi-turn injection, it has apertures of $272 \pi \mathrm{mm}$ mrad and $49 \pi \mathrm{mm} \mathrm{mrad}$ in the horizontal and vertical planes, respectively [2]. This vertical emittance growth becomes crucial as the beam intensity increases. Since the acceleration period is rather short, we chose the BEAMSCOPE method [3], which can measure a beam profile in $100 \mu \mathrm{s}$. It is difficult to use a wire scanner and other methods for a quick measurement. In order to measure the beam profile exactly, a precise calibration was performed [1].

\section{MEASUREMENT}

The beam-profile measurement system comprises a beam scraper and two pulsed bump magnets which kick the beam in the vertical direction. Figure 1 shows a plan view of the Booster synchrotron. Vertical bump magnets are installed in straight sections S2 and S4. Since the phase advance between the magnets is $\pi$ radians and the beta functions at both magnets are same, a bump orbit is formed from S2 to S4 in the vertical plane. The magnets are connected in series and excited by a pulsed current having the same polarity. The pulse shape is a half-sine and the pulse width is $200 \mu \mathrm{s}$. M1-M8 are alternating-gradient magnets and have a focusing order of FDDF. The beam scraper is located in straight section S3, where the bump orbit has its extremum.

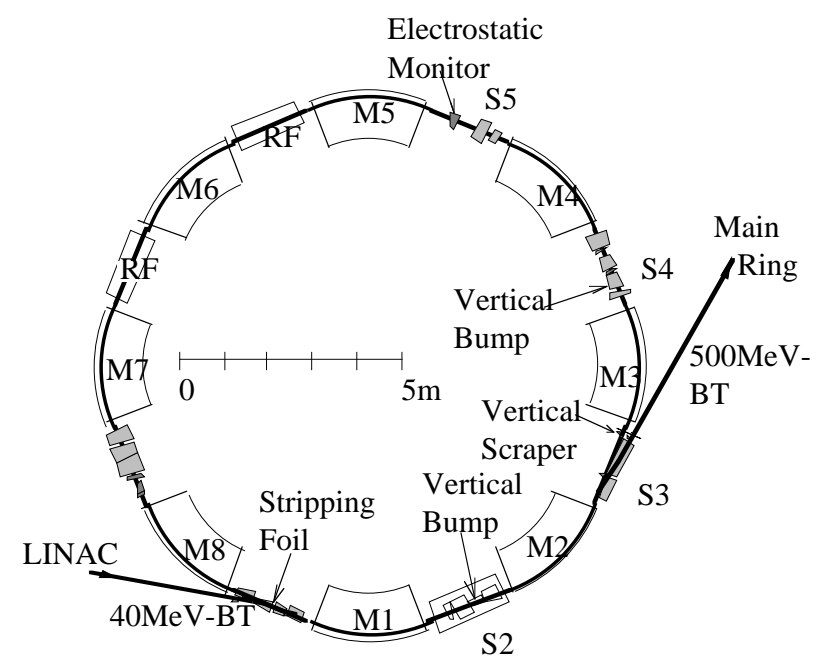

Fig. 1. Plan of the Booster synchrotron.

The ratio of the current of the bump magnets and the height of bump orbit at the scraper was precisely calibrated by means of a pencil beam, which was much smaller than the ring acceptance. In order to measure the beam profile exactly, it is essential to calibrate the bump current to the beam displacement with good accuracy. The ratio is reproducible and independent of the beam parameters, except for the energy. The beam scraper was movable and located at the optimum position where the aperture was minimum in the ring, but did not disturb the circulating beam. The current of the bump magnets was optimized to be able to scrape the whole beam as slowly as possible. Figure 2 shows a schematic view of the beam-profile measurement system. The current of the bump magnets and the beam-bunch signal were recorded simultaneously using a digital sampling oscilloscope, which records the all data during $200 \mu$ s with a sampling rate of $200 \mathrm{MHz}$. The current of the bump magnets and bunch signal were measured by a current transformer and a fast electrostatic monitor (ESM), respectively. 


\section{SPACE CHARGE TUNE SHIFT}

The Booster tune space is shown in Fig. 6. In order to estimate the space charge detuning in the smooth approximation for $\Delta \mathrm{p} / \mathrm{p}=0$, we use the expression [4]

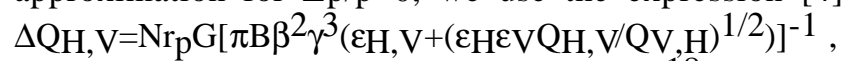
where $\mathrm{N}=$ number of protons, $\mathrm{r}_{\mathrm{p}}=1.535 * 10^{-18} \mathrm{~m}$, and $\mathrm{G}$ is a form factor which depends on the transverse intensity distribution. For $1.0^{*} 10^{12} \mathrm{ppp}$ at the end of injection, where $\beta^{2} \gamma^{3}=0.09$ and the bunching factor is $\sim 1$, the $90 \%$ beam width was $14 \mathrm{~mm}$. Using $\mathrm{G}=1.56$ for the Gaussian distribution, we obtain $\Delta \mathrm{Q}_{\mathrm{ysc}}=-0.10$ and $\Delta \mathrm{Q}_{\mathrm{xsc}}=-0.05$, as shown in Fig. 6(a), bounded by the bold line. At $1 \mathrm{~ms}$ after injection, where emittance growth has occurred for the beam core but not for the beam tail, the beam is captured in the RF bucket and the bunching factor becomes 0.3 . Then, $\Delta \mathrm{Q}_{\mathrm{ysc}}=-0.34$ and $\Delta \mathrm{Q}_{\mathrm{xsc}}=-0.17$ are obtained for the same beam widths. This is the lowest point of the region in Fig. 6(a) bounded by the solid line, and touches both $\mathrm{Qx}=2$ and $\mathrm{Qy}=2$. For $1.66^{*} 10^{12} \mathrm{ppp}$ at the end of injection, we obtain $\Delta \mathrm{Q}_{\mathrm{ysc}}=-0.17$ and $\Delta \mathrm{Q}_{\mathrm{xsc}}=-0.08$, as shown in Fig. 6(b), bounded by the bold line. For bunched beam showing emittance growth for both the beam core and edge, the $90 \%$ beam width became $18 \mathrm{~mm} 1 \mathrm{~ms}$ after injection. We obtain $\Delta \mathrm{Q}_{\mathrm{ysc}}=-0.40$ and $\Delta \mathrm{Q}_{\mathrm{xsc}}=-0.26$ which cross both $\mathrm{Qx}=2$ and $\mathrm{Qy}=2$ resonances largely, as shown in Fig. 6(b).

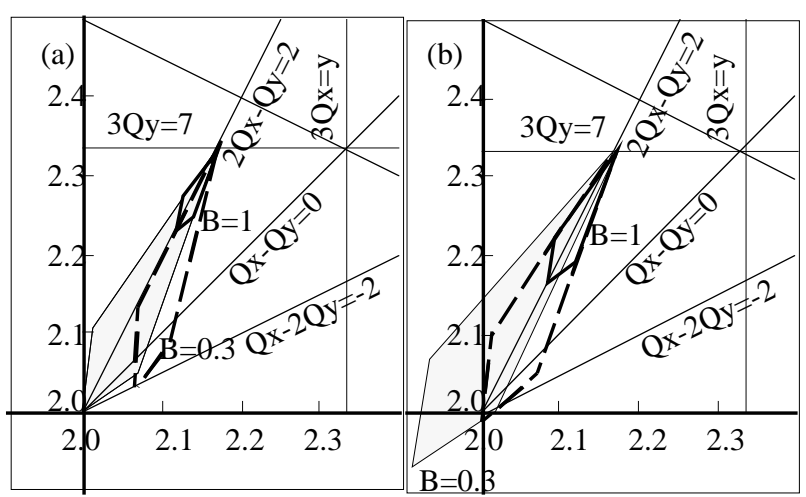

Fig. 6 KEK-PS Booster Tune Spaces at $1.0 * 10^{12}$ ppp (a) and $1.66 * 10^{12} \mathrm{ppp}(\mathrm{b})$.

However, the real beam is not smooth and $\Delta \mathrm{p} / \mathrm{p} \neq 0$. For a more accurate estimation we used the computer code space SPACEX [5], which can solve envelope equations including the space charge force. The results are also shown in Fig. 6. We assume that the momentum spread was $0.30 \%$ and that the real beam was larger in the horizontal plane due to it. Using $\mathrm{G}=1.56$ and $\mathrm{B}=0.3$, we obtain $\Delta \mathrm{Q}_{\mathrm{ysc}}=-0.30$ and $\Delta \mathrm{Q}_{\mathrm{Xsc}}=-0.11$ for $1 * 10^{12} \mathrm{ppp}$. It is shown in Fig. 6(a), bounded by the dashed line. We also obtained $\Delta \mathrm{Q}_{\mathrm{ysc}}=-0.34$ and $\Delta \mathrm{Q}_{\mathrm{xsc}}=-0.17$ for $1.66 * 10^{12}$ ppp. This is shown in Fig. 6(b), bounded by the dashed line. Since the momentum spread was taken into account, the tune spreads obtained by SPACEX are smaller than those by Laslett formula. Since the $90 \%$ beam width during the emittance growth was smaller than $18 \mathrm{~mm}$, the tune spread should have been much larger than the region in Fig. 6(b) bounded by the dashed line. It is reasonable that the large tune spread which crosses the integer and other resonances causes emittance growth, which stops when the tune spread becomes smaller.

\section{DISCUSSIONS}

The emittance growth in the vertical plane can be explained based on the space charge effects. Operation at higher tunes and resonance correction techniques are used to avoid and overcome the effects. However, the Booster synchrotron is a small accelerator and has limited space. It is difficult to install the necessary instruments to change the tunes and for corrections. Reduction of the peak intensity by the second-harmonic buncher is another way, and might be possible if the instrument is designed to be compact.

\section{REFERENCES}

[1] T. Adachi et al., "Beam Profile Measurement in the KEK-PS Booster Using Pulsed Bump Magnets and Movable Scraper", Proceedings of the 4th European Particle Accelerator Conference (EPAC94), London, U.K., July 1994.

[2] T. Suzuki, "ORBIT ANALYSIS OF THE KEK SYNCHROTRON", KEK-74-4.

[3]H. Shonauer, "Experience with the BEAMSCOPE Emittance Measurement System at the CERN-PS Booster", Proceedings of the Workshop on Advanced Beam Instrumentation, KEK, Tsukuba, Japan, April 1991, pp. 453-466.

[4] L. J. Laslett, BNL Summer Study, BNL 7534, pp. 324-67(1963).

[5] G. Rees, private communications. 


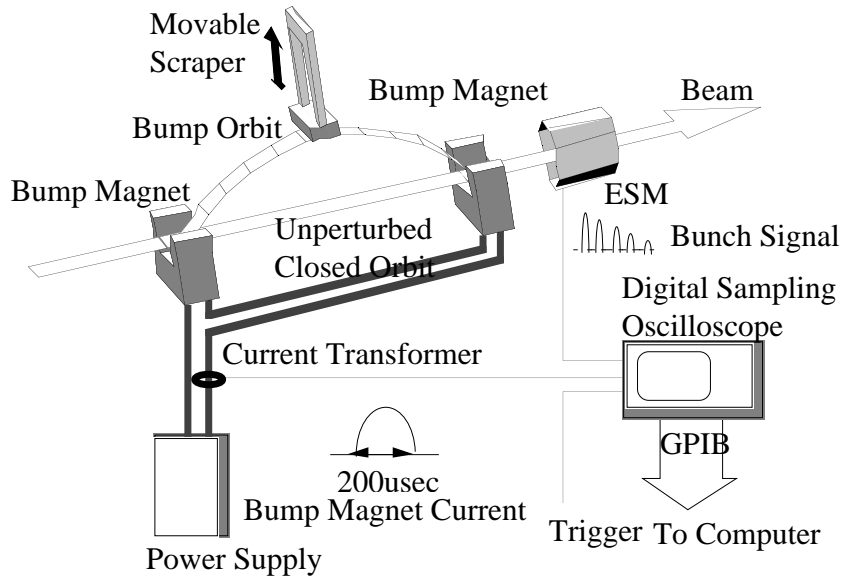

Fig. 2. Schematic of the profile measuring system.

We measured the intensity dependence of the beam profiles in the vertical plane for intensities from $1.8^{*} 10^{11}$ to $2.2 * 10^{12}$ particle per bunch (ppp). Since we wanted to use the same initial beam distributions for the different beam intensities, a beam slit of mesh shape was installed at the entrance of the $40-\mathrm{MeV}$ Linac. Figures 3 and 4 show the normalized emittance containing $90 \%$ of the beam and normalized $1-\sigma$ emittance, respectively, against a delay time of up to $5 \mathrm{~ms}$ from injection for various intensities. In these figures, the emittance at injection, i.e. Delay Time $=0$ $\mathrm{ms}$, was obtained from the beam profile taken by moving the beam scraper without exciting the bump magnets, since the ESM cannot be available during injection where a bunch structure has not been sufficiently formed. The initial beam emittances are constant for different intensities for both $90 \%$ and $1-\sigma$ emittance (Figs. 3 and 4 ). The initial distribution was independent of the beam intensity. Figures 3 and 4 also show the emittance growth which occurred before $\sim 1 \mathrm{~ms}$.

The emittance growth is clearly observed at intensities larger than $1.66 * 10^{12}$ ppp in Fig. 3. The emittance growth between intensities of $1.4 * 012$ ppp and $1.66 * 10^{12} \mathrm{ppp}$ is much larger than that between intensities of $1.66 * 10^{12} \mathrm{ppp}$ and $2.2 * 10^{12} \mathrm{ppp}$. However, we should note that there might be a small amount of beam loss for $2.2 * 10^{12}$ ppp. The emittance growth is observed at intensities larger than $1 * 10^{12} \mathrm{ppp}$. The normalized $1-\sigma$ emittance becomes larger according the increment of the beam intensity. The threshold intensity for $1-\sigma$ emittance is about $40 \%$ less than that for $90 \%$ emittance. Figure 5 shows the FWHM and the width which contains $90 \%$ of the beam against the beam intensities. It clearly shows that the intensities which cause the emittance growths are different for the beam core and edge. The difference of the threshold intensities for emittance growths suggests that it is caused by space charge effects.

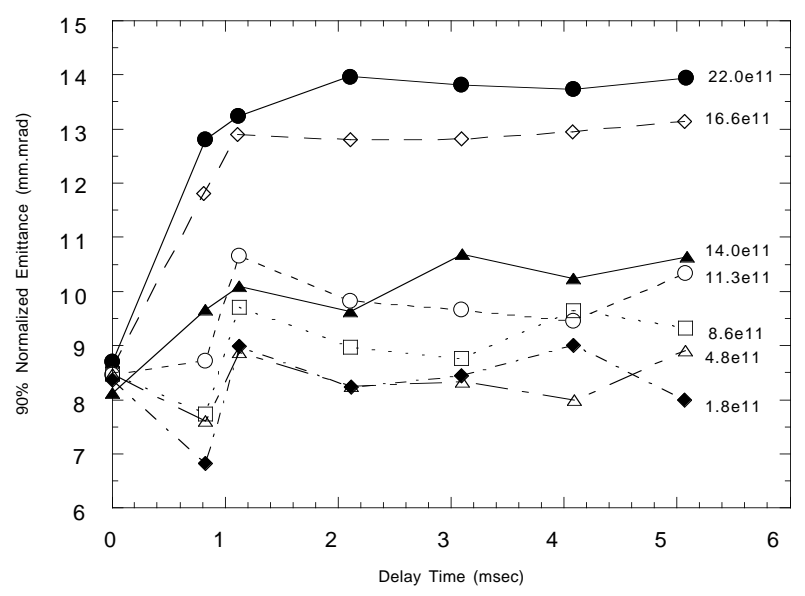

Fig. 3. Intensity dependence of the normalized emittance which contains $90 \%$ of the beam.

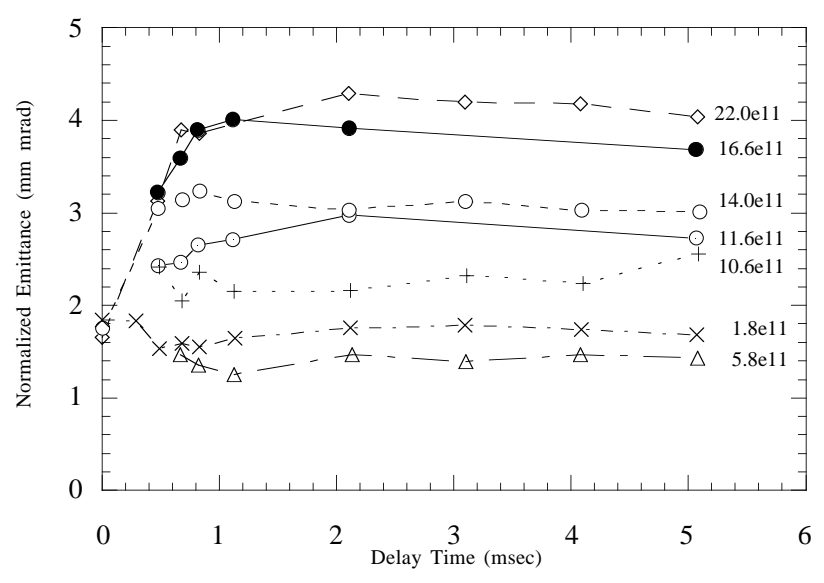

Fig. 4. Intensity dependence of the normalized 1$\sigma$ emittance.

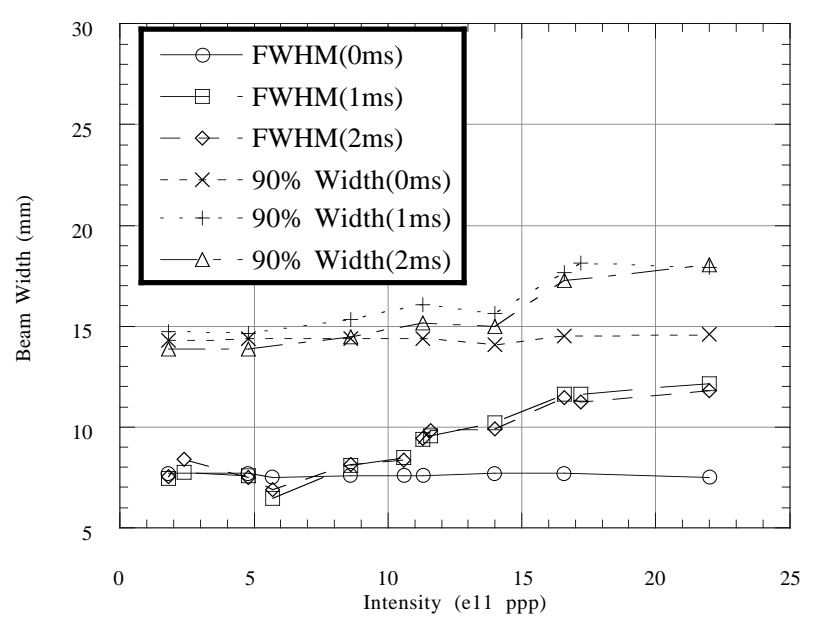

Fig. 5. Intensity dependence of the beam widths. 